\title{
Phase congruency features for palm-print verification*
}

\author{
V. Štruc, N. Pavešić \\ Faculty of Electrical Engineering \\ University of Ljubljana, Tržaška 25, SI-1000 Ljubljana, Slovenia \\ vitomir.struc@fe.uni-lj.si,nikola.pavesic@fe.uni-lj.si
}

\begin{abstract}
The existing palm-print verification schemes have demonstrated good verification performance when identity claims have to be verified based on palm-print images of adequate quality (e.g., acquired in controlled illumination conditions, free from distortions caused by the pressure applied to the surface of the scanner, etc.). However, most of these schemes struggle with their verification performance when features have to be extracted from palm-print images of a poorer quality. In this paper we present a novel palm-print feature-extraction approach which deals with the presented problem by employing the $2 D$ phase congruency model for line-feature-extraction. The proposed approach first computes a set of phase congruency features from a palm-print image and subsequently performs linear discriminant analysis on the computed features to represent them in a more compact manner. The approach was tested on two contrasting databases, namely, on the FE-LUKS and on the PolyU database. Encouraging results were achieved on both databases.
\end{abstract}

\section{Introduction}

The widespread deployment of information technology during the last decades with its e-banking, e-government or health monitoring applications has triggered the need for new authentication (or verification) schemes that could replace the traditionally employed and easily compromisable token- (e.g., identification cards) and knowledge-based (e.g., passwords or personal identification numbers) means of identification $[1,2,3]$. The current trend in meeting this need is to design biometric security systems which exploit the physical and/or behavioral attributes of an

\footnotetext{
* Portions of this work have been tested on the PolyU Palmprint Database
} 
individual to establish identity. As this attributes are unique to each individual and cannot be forgotten, stolen or lost, they hold a great potential in replacing the conventional token- and knowledge-based security schemes.

Many biometric systems have already been presented in the literature; among them, systems which employ biometric traits, such as fingerprints, face, voice, iris, retina, hand-geometry, signature or palm-prints, are the most common [4]. Each of the listed biometric characteristics has its own strengths and weaknesses and is consequently more or less suited for a particular application domain. Face- and voice-based recognition systems, for example, are considered to be unintrusive, but they do, however, still have problems achieving high recognition accuracy, especially when biometric samples (i.e., face images or speaker recordings) are captured in uncontrolled environments. Iris and retinal recognition, on the other hand, exhibit high recognition accuracy, but require intrusive acquisition systems [5]. Opposed to these recognition systems, palm-print based recognition is considered both user-friendly as well as fairly accurate and thus provides an attractive alternative to other biometric systems.

The existing palm-print recognition systems can, according to [6], (based on the employed feature-extraction technique) be classified into one of three groups: texture- (e.g., [7, 8]), line- (e.g., $[9,10,11,12])$ and appearancebased systems (e.g., $[13,14,15,16])$. Though all feature types are relevant for palm-print-based biometric recognition, this paper focuses on line-based features.

Most of the palm-print recognition systems that make use of line features to verify the identity of a user employ gradient-based methods to extract characteristic lines from a palm-print image (e.g., [13, 14]). While these methods work fine on images of an appropriate quality (e.g., acquired in controlled illumination conditions, free of distortions caused by the pressure applied to the surface of the scanner, etc.), they have problems when features have to be extracted from palm-print images of a poorer quality. In these situations a more robust approach is preferable. To this end, we have developed a novel palm-print verification approach which uses line features extracted with the phase congruency model and is, therefore, relatively insensitive to image distortions caused by the acquisition procedure (note that images acquired with a desktop scanner almost always contain regions distorted by pressure; similarly, unideal illumination during the image acquisition stage also causes distortions on the palm-print image when a camera is used for image capturing - see Fig. 1).

The rest of the paper is organized as follows: Section 2 gives a short description of the basic characteristics of a palm-print recognition system. In Section 3 the novel phase congruency based feature-extraction technique is introduced. Section 4 presents the matching procedure and classification rule used for verifying identity claims based on the proposed approach. The two palm-print databases employed in our experiments and their associated experimental protocols are described in Section 5. The experiments are presented in Section 6, while the paper concludes with some final remarks and directions for future work in Section 7. 


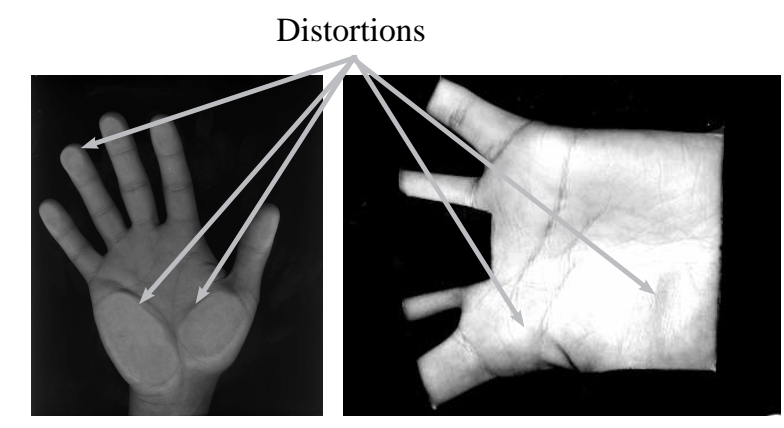

Figure 1. Distortions of a palm-print image: acquired with a desktop scanner (left), acquired with a CCD camera (right)

\section{On palm-print verification systems}

Palm-print verification systems, like most biometric systems, are comprised of the following five modules [17, 18]: (i) the data acquisition or sensor module, which captures the biometric data, i.e., the image of the hands palmar surface, of the person currently presented to the system; (ii) the pre-processing module that extracts the region of interest (ROI), i.e., the palm-print region, from the acquired image data and normalizes the extracted ROI in respect to size, rotation and illumination; (iii) the feature-extraction module, which computes a set of representative and discriminative features from the extracted and normalized palm-print ROI; (iv) the matching and decision making module that first compares the computed feature set with a template, i.e., the mathematical representation of the feature sets extracted during the enrollment session, and outputs a similarity (or matching) score which is then used to decide whether the person presented to the system is who he/she claims to be; and $(v)$ the system's database module, which is used by the palm-print verification system for storing the templates of the enrolled users [2]. The block diagram of the described palm-print verification system is shown in Fig 2.

Here Fig. 2a depicts the enrollment stage during which multiple palm-print samples of a user are acquired, transformed into a feature space, represented in the form of a client template, associated with the user's identity and ultimately stored in the system's database. Fig. 2b, on the other hand, shows the verification stage during which the identity claim of the person presented to the palm-print verification system is either verified or rejected.

\section{Feature extraction using phase congruency}

Let us for the moment assume that the palm-print images have already been properly pre-processed, i.e., extracted from the acquired images and appropriately normalized, and are, thus, suitable for feature-extraction. Such an assumption is reasonable due to the fact that the pre-processing procedure of the palm-print verification system depends heavily on the intrinsic characteristics of the image acquisition procedure (e.g., the presence of 


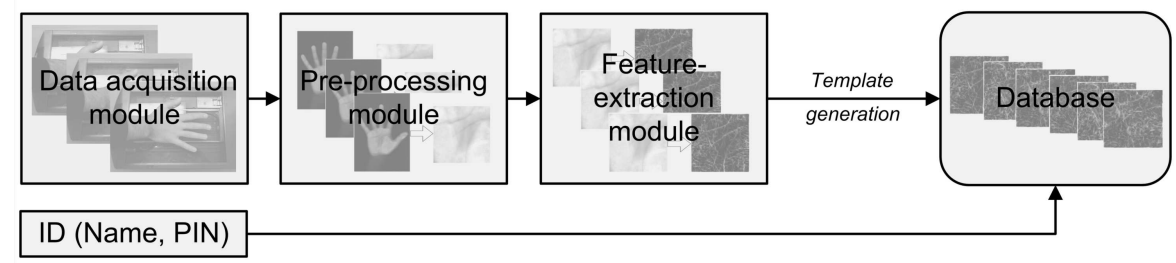

(a)

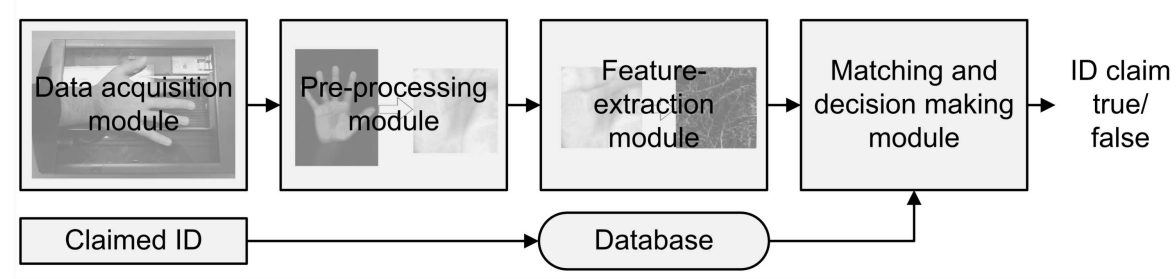

(b)

Figure 2. The block diagram of a palm-print verification system

guiding pegs, the visibility of fingers, etc.). Thus, the palm-print pre-processing is linked to the characteristics of the database used for evaluating one's recognition techniques and will, in our case, be described in Sections 5.1 and 5.2 where the two databases employed in our experiments are presented. In this section, however, we will present the novel line-feature-extraction technique which first extracts a set of phase congruency features from the input palm-print image and then applies LDA on this feature set to represent the phase congruency features in a discriminative and compact manner.

\subsection{Phase congruency features}

There have been a number of palm-print recognition systems presented in the literature that make use of linebased features, e.g., $[9,10,19]$. Typically, these systems use line detectors, such as the Sobel operator, which scan the palmprint image for points of high intensity gradients to extract the line-features. However, varying illumination conditions during the image acquisition stage (when images are captured with a camera-based sensor) or distortions of the palm-print region caused by the pressure applied to the surface of the scanner (when images are recorded using an optical scanner) often result in the detection of spurious lines. To avoid the presented difficulties, we propose to employ the phase congruency model for line-feature-extraction.

Rather than searching for points of high intensity gradients, the model seeks those points in the palm-print ROI where the 2D log-Gabor filter responses (of the palm-print ROI) over several scales and orientations are maximal in phase $[20,21]$. Thus, a point in an image is of significance only if the phase responses of the log-Gabor filters over a range of scales and orientations display some kind of order. As pointed out by Kovesi in [20], phase congruency acts as a line (or edge) descriptor of an image and is, unlike gradient-based edge-detectors, such as 
those developed by Sobel [22], Marr and Hildreth [23] or Canny [24], not susceptible to image variations caused by illumination, blurring, magnification and alike. Its robustness to image variations stems from the multi-scale and multi-orientational approach to phase congruency calculation as well as from the fact that phase rather than magnitude information is considered for line (or edge) detection ${ }^{1}$. Clearly, the phase congruency model exhibits desirable characteristics, which should prove useful when deriving (robust) line-features from palm-print ROIs.

Let $\mathcal{G}=\left\{G\left(f_{h}, \theta_{g}\right): h=1,2, \ldots, p ; g=1,2, \ldots, r\right\}$ denote the set of $2 \mathrm{D}$ log-Gabor filters with $p$ scales and $r$ orientations and let $G\left(f_{h}, \theta_{g}\right)=G_{h g}$ be defined as:

$$
G_{h g}=\exp \left\{\frac{-\left[\ln \left(f / f_{h}\right)\right]^{2}}{2\left[\ln \left(k / f_{h}\right)\right]^{2}}\right\} \exp \left\{\frac{-\left(\theta-\theta_{g}\right)^{2}}{2 \sigma_{\theta}^{2}}\right\}
$$

where $f$ and $\theta$ denote the polar coordinates of the log-Gabor filter in the frequency domain, $f_{h}$ denotes the filter's center frequency (in our experiments it was set to $f_{h}=0.33 \cdot(2.1)^{1-h}$ ), $k$ defines the bandwidth of the filter in the radial direction (the ratio $k / f_{h}$ is commonly set to a constant value, for example 0.55 , as in our case), $\theta_{g}=(g-1) \cdot \pi / r$ represents the orientation of the filter and $\sigma_{\theta}$ controls the angular bandwidth of the 2D log-Gabor filter (we used a value of $\sigma_{\theta}=1.2 \cdot(\pi / r)$ ).

Furthermore, let $I(x)$, where $x$ stands for the pixel location in the spatial domain, denote the grey-scale distribution of the palm-print image. The magnitude $A_{h g}(x)$ and phase $\phi_{h g}(x)$ responses of the image $I(x)$ at a specific scale $h$ and orientation $g$ of the log-Gabor filter can then be computed as:

$$
\begin{aligned}
& A_{h g}(x)=\sqrt{\operatorname{Re}^{2}\left[I(x) * G_{h g}^{s}\right]+\operatorname{Im}^{2}\left[I(x, y) * G_{h g}^{s}\right]}, \\
& \phi_{h g}(x)=\arctan \left(\operatorname{Im}\left[I(x) * G_{h g}^{s}\right] / \operatorname{Re}\left[I(x) * G_{h g}^{s}\right]\right),
\end{aligned}
$$

where * denotes the convolution operator, $G_{h g}^{s}$ stands for the log-Gabor filter in the spatial domain at the scale $h$ and the orientation $g$ and $\operatorname{Re}[X]$ and $\operatorname{Im}[X]$ represent the real and imaginary parts of the convolution output.

Finally, the two-dimensional phase congruency features can according to [20] be computed using the following expression:

$$
P C_{2 D}(x)=\frac{\sum_{g} \sum_{h} W_{g}(x)\left\lfloor A_{h g}(x) \Delta \Phi_{h g}(x)-T_{g}\right\rfloor}{\sum_{g} \sum_{h} A_{h g}(x)+\varepsilon},
$$

where $T_{g}$ represents the estimated noise energy at orientation $g, W_{g}(x)$ denotes a weighing function that weighs for the frequency spread, $\varepsilon$ is a small constant which prevents divisions by zero, the symbols \lfloor\rfloor denote the following

\footnotetext{
${ }^{1}$ For a detailed analysis of the phase congruency model, its characteristics and advantages over gradient-based edge-detectors the reader should refer to $[20]$
} 
operation:

$$
\lfloor X-T\rfloor= \begin{cases}X-T & , \text { if } X>T \\ 0 & , \text { otherwise }\end{cases}
$$

and $\Delta \Phi_{h g}(x)$ is a phase deviation measure defined as:

$$
\Delta \Phi_{h g}(x)=\cos \left(\phi_{h g}(x)-\bar{\phi}_{g}(x)\right)-\left|\sin \left(\phi_{h g}(x)-\bar{\phi}_{g}(x)\right)\right| .
$$

In equation (6) $\phi_{h g}(x)$ denotes the phase angle at the location $x$ of the log-Gabor filter phase response at scale $h$ and orientation $g$, while $\bar{\phi}_{g}(x)$ represents the mean phase angle at the orientation $g$.

Once a palm-print image is transformed using the described phase congruency model, the final feature vector $\mathbf{x}$ is constructed by dividing the phase congruency image into a number of non-overlapping blocks of size $2 \times 2$ pixels and then computing the mean value and standard deviation of the pixels in each of the $m$ blocks, i.e.,

$$
\mathbf{x}=\left(\mu_{1}, \sigma_{1}, \mu_{2}, \sigma_{2}, \ldots, \mu_{m}, \sigma_{m}\right)^{T}
$$

It has to be noted that the final feature vector $\mathbf{x}$ could also be constructed by a simple concatenation of the elements/pixels comprising the phase congruency image. The described approach, which calculates the $2 \times 2$ block means and standard deviations, was employed only to ensure equal footing with the second line-feature technique tested in Section 6.2, as it also uses the presented methodology for constructing the palm-print feature vector.

However, as we can see from Fig. 3, the line features extracted with the phase congruency model vary in their appearance when log-Gabor filters with different numbers of scales and orientations are used. The effects of these parameters on the verification performance of the proposed feature-extraction technique will be assessed in Section 6.1 .

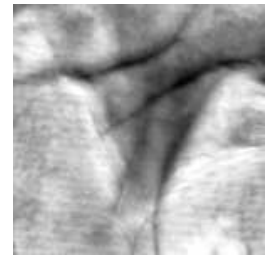

(a)

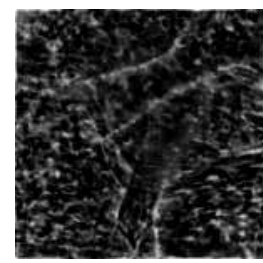

(b)

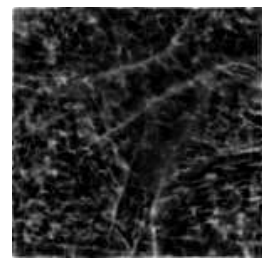

(c)

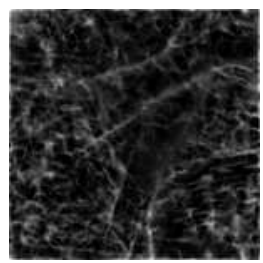

(d)

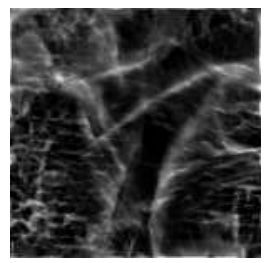

(e)

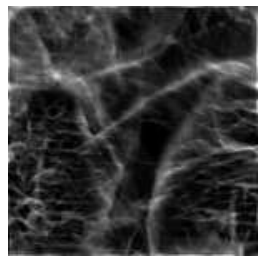

(f)

Figure 3. a) A normalized palm-print image; Phase congruency image for $\mathbf{b}) p=3$ and $r=4$, c) $p=3$ and $r=6, \mathbf{d}) p=3$ and $r=8, \mathbf{e )} p=5$ and $r=6, \mathbf{f}) p=5$ and $r=8$ 


\subsection{Linear discriminant analysis}

Let us consider a set of $n d$-dimensional (where $d=2 m$ ) training phase congruency feature vectors $\mathbf{x}_{i}$ arranged in a $d \times n$ column data matrix $\mathbf{X}$, i.e., $\mathbf{X}=\left[\mathbf{x}_{1}, \mathbf{x}_{2}, \ldots, \mathbf{x}_{n}\right]$, and let us assume that each of these feature vectors stems from one of the $N$ classes, i.e., subjects, labeled as $\omega_{1}, \omega_{2}, \ldots, \omega_{N}$. Based on the training data comprising the matrix $\mathbf{X}$, LDA tries to achieve maximum class-separation by maximizing Fisher's discriminant criterion, which is defined as the ratio of the between-class to the within-class scatter matrix [13], i.e.,

$$
J(\mathbf{W})=\frac{\left|\mathbf{W}^{T} \boldsymbol{\Sigma}_{B} \mathbf{W}\right|}{\left|\mathbf{W}^{T} \boldsymbol{\Sigma}_{W} \mathbf{W}\right|}
$$

Here, the between-class and within-class scatter matrices $\boldsymbol{\Sigma}_{B}$ and $\boldsymbol{\Sigma}_{W}$ are defined as:

$$
\begin{gathered}
\boldsymbol{\Sigma}_{B}=\sum_{i=1}^{N} n_{i}\left(\boldsymbol{\mu}_{i}-\boldsymbol{\mu}\right)\left(\boldsymbol{\mu}_{i}-\boldsymbol{\mu}\right)^{T}, \\
\boldsymbol{\Sigma}_{W}=\sum_{i=1}^{N} \sum_{\mathbf{x}_{j} \in \omega_{i}}\left(\mathbf{x}_{j}-\boldsymbol{\mu}_{i}\right)\left(\mathbf{x}_{j}-\boldsymbol{\mu}_{i}\right)^{T},
\end{gathered}
$$

and the symbols $\boldsymbol{\mu}, \boldsymbol{\mu}_{i}$ and $n_{i}$ denote the grand mean of all training feature vectors, the $i$-th class conditional mean and the number of feature vectors in the $i$-th class, respectively.

The result of the maximization procedure is the LDA transformation matrix $\mathbf{W}$ which can be shown to consist of the $d^{\prime}<N-1$ leading eigenvectors of the following eigenproblem:

$$
\boldsymbol{\Sigma}_{W}^{-1} \boldsymbol{\Sigma}_{B} \mathbf{w}_{i}=\lambda_{i} \mathbf{w}_{i}, \quad i=1,2, \ldots, d^{\prime} .
$$

By using the calculated transformation matrix $\mathbf{W}=\left[\mathbf{w}_{1}, \mathbf{w}_{2}, \ldots, \mathbf{w}_{m}\right]$ an arbitrary phase congruency feature vector $\mathbf{x}$ centered around the grand mean $\boldsymbol{\mu}$ can be projected into the LDA subspace with the help of the following expression:

$$
\mathbf{y}=\mathbf{W}^{T}(\mathbf{x}-\boldsymbol{\mu})
$$

thus reducing the vectors dimensionality from $d$ to $d^{\prime}$.

In the field of palm-print recognition, however, the number of available training samples (i.e., training phase congruency feature vectors) per class is usually significantly smaller than the number of elements contained in each sample. This fact renders the matrix $\boldsymbol{\Sigma}_{W}$ singular (its' rank is at most $n-N$ ) and the computation of the transformation matrix $\mathbf{W}$ using equation (11) impossible. To overcome this problem, we first projected the matrices $\boldsymbol{\Sigma}_{B}$ and $\boldsymbol{\Sigma}_{W}$ into the principal component subspace to ensure that the matrix $\boldsymbol{\Sigma}_{W}$ is nonsingular and 
thus invertible. LDA was then performed in the new subspace. A detailed description of the employed approach can be found in [13].

\section{The matching procedure and classification rule}

In a palm-print recognition system operating in verification mode the feature vector $\mathbf{y}$ extracted from the given input palm-print image by the procedure described in the previous section is compared (or matched) with the template $\overline{\mathbf{y}}_{i}$ associated with the claimed identity [25]. Here, the template $\overline{\mathbf{y}}_{i}$ is constructed by averaging all feature vectors of the $i$-th client available during the enrollment stage. The following similarity measure is used to produce the matching score:

$$
\delta_{\cos }\left(\mathbf{y}, \overline{\mathbf{y}}_{i}\right)=\frac{\mathbf{y} \cdot \overline{\mathbf{y}}_{i}}{|\mathbf{y}|\left|\overline{\mathbf{y}}_{i}\right|}
$$

where the operator - denotes the dot product.

If the value of the cosine similarity measure defined by (13) is higher than a pre-defined decision threshold, then the claim of identity is classified as being genuine (or authentic), otherwise the claim is classified as being false (or unauthentic).

\section{$5 \quad$ Databases and experimental setups}

The effectiveness of the proposed phase-congruency-based feature extraction technique was assessed on two palm-print databases, namely, the FE-LUKS and the PolyU database. The databases exhibit some fundamental differences among which the image acquisition procedure is probably the most important - the FE-LUKS database was collected using a desktop scanner, while the PolyU database employed a CCD camera to record images of the palmar surface of the hand. A more detailed description of both databases will be given in the remainder of this section.

\subsection{The FE-LUKS database}

The FE-LUKS database [17] was acquired at the Laboratory of Artificial Perception, Systems and Cybernetics of the Faculty of Electrical Engineering in Ljubljana (Slovenia). It contains 2000 hand images that correspond to 200 distinct subjects. Each subject in the database is thus represented with a total of 10 hand-images. The images (with 256 grey levels) were recorded with the help of a commercial desktop scanner rated at a resolution of 180 dots per inch. Some examples of the hand-images from the FE-LUKS database are shown in Fig. 4.

The presented sample images clearly show the level of distortion of the hands' palmar surface due to the characteristics of the image acquisition procedure. These distortions, however, should in an ideal case have no 

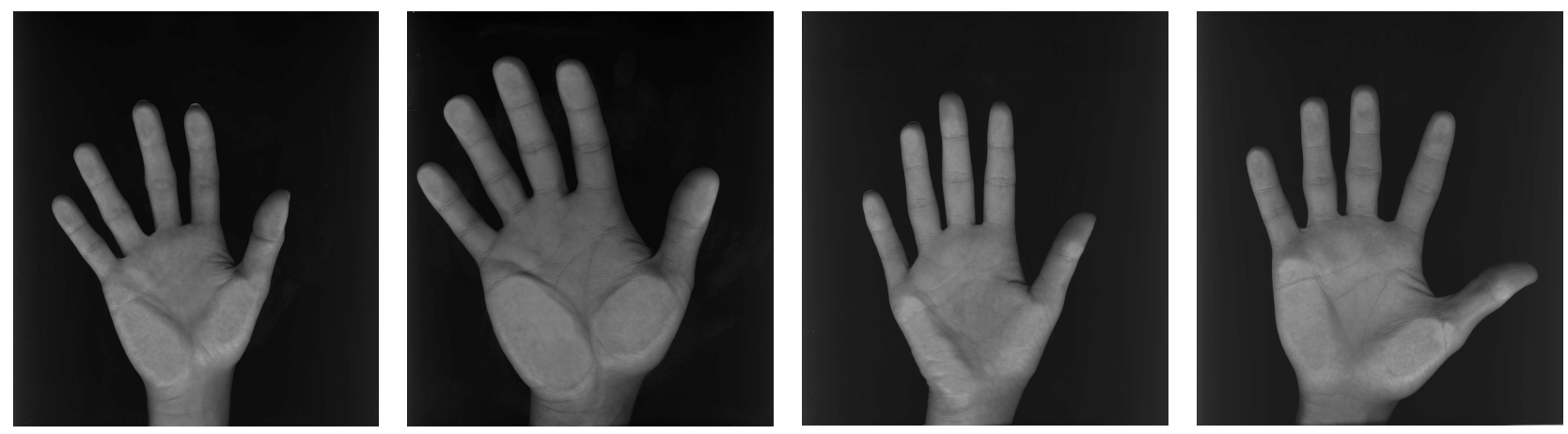

Figure 4. Sample images from the FE-LUKS database

impact on the performance of a palm-print verification system.

Images from the FE-LUKS database contain the whole palmar surface of the hand. Hence, for the palm-print verification experiments the images have to be subjected to a pre-processing procedure which extracts and normalizes the region of interest (ROI), i.e., the palm-print region, from the original hand-images. Such a segmentation step is common to most biometric systems. In face recognition systems, for example, it includes the extraction of the facial region from an image [25], in speaker recognition systems it involves the detection of speech and non-speech segments [26] from a speaker recording and so forth. In our experiments a pre-processing procedure comprising of the following steps was employed [17, 27]: (i) binarization - in the first step a binary mask (Fig. 5b) of the hand region was extracted from the grey-scale hand image (Fig. 5a) using an image thresholding procedure, (ii) contour extraction - in the second step the contour (Fig. 5c) of the hand was extracted from the binary hand-region mask, (iii) ROI localization - in the third step first the positions of the local minima and maxima of the hand contour were determined and then the ROI was located as shown in Fig. 5d, (iv) normalization in the last step the final palm-print ROI (Fig. 5e) was obtained by rotating the cropped palm-print region to a pre-defined orientation and resizing it to the fixed size of $64 \times 64$ pixels; prior to feature extraction the photometric normalization procedure which featured a conversion of the pixel intensity distribution to $\mathcal{N}(0,1)$ was also applied to the geometrically normalized palm-print ROI.

\subsection{The PolyU database}

The second database used in our experiments is the PolyU database. The database was acquired at the Hong Kong Polytechnic University (China) using a CCD camera [28, 29]. In its current version the database contains 7752 (8-bit) grey-scale images corresponding to 386 subjects. Unlike the FE-LUKS database, where the number of images per subject is fixed, each subject in the PolyU database is accounted for with approximately 20 images. The exact number of images per subject, however, varies from case to case. The hand-images were captured in two separate sessions; the average interval between the first and the second session being two months. From the sample 


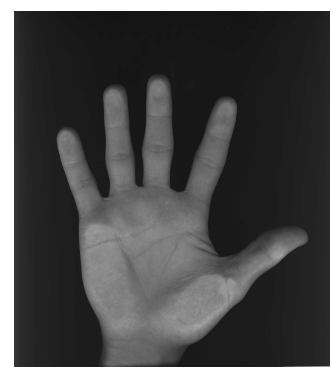

(a)

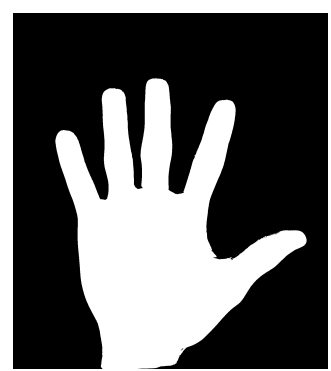

(b)

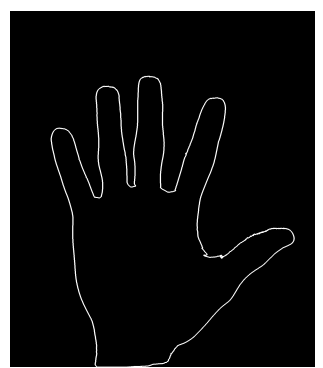

(c)

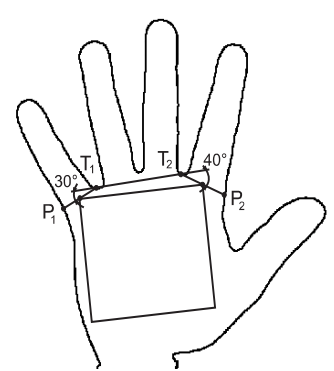

(d)

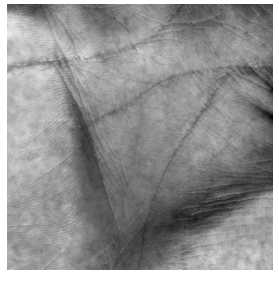

(e)

Figure 5. The pre-processing procedure of the FE-LUKS database: (a) a hand image from the FELUKS database, (b) the binary image of the hand region, (c) the image of the contour of the hand region, (d) extraction of the palm-print ROI, (e) the normalized palm-print image

palms of the PolyU database presented in Fig. 6 we can see that the palmar surface of the hands is uniformly illuminated ${ }^{2}$. Therefore, no distortions are visible on the palm-prints as in the case of the FE-LUKS database hand-images.
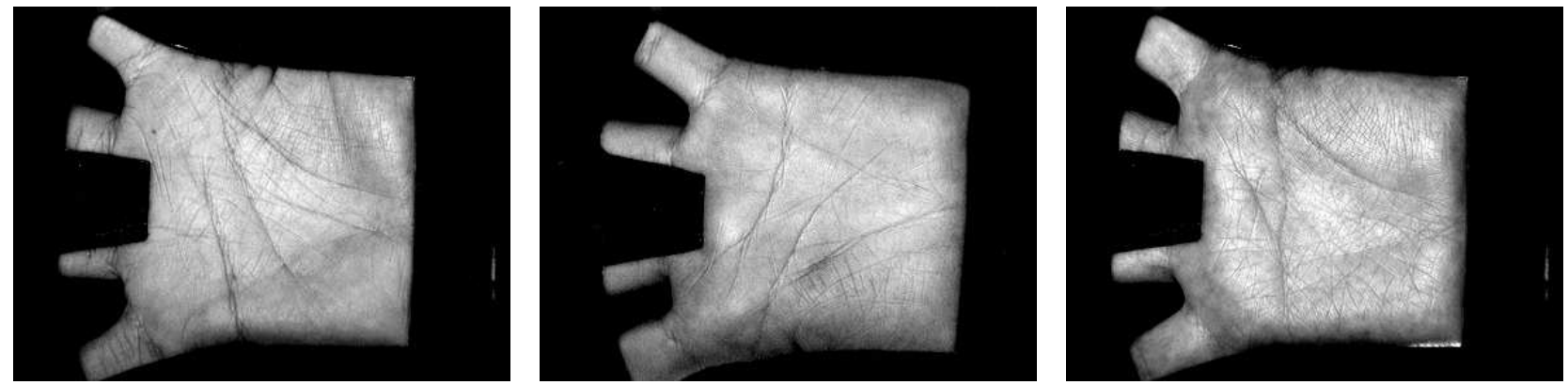

Figure 6. Sample images from the PolyU database

The PolyU database was captured with a specially developed palm-print acquisition system [28] which consequently defines the appearance of the recorded hand-images (e.g., there are no finger tips, no thumb, etc.). Based on the image characteristics Zhang et al. [13] proposed a pre-processing procedure to be used with the PolyU database. The procedure which was also employed in our experiments consisted of: (i) a contour extraction algorithm that followed the same steps as described in Section 5.1, i.e., binarization and contour extraction (Fig. 7 a-c), (ii) a routine that computed a coordinate system based on the gaps between the upper two and the lower two fingers (Fig. 7d) and (iii) a normalization procedure that, based on the determined coordinate system, cropped the palm-print ROI to a size of $128 \times 128$ and subsequently normalized the cropped region in the same way as it was done with the hand-images from the FE-LUKS database (Fig. 7e), i.e., geometrically and photometrically.

\footnotetext{
${ }^{2}$ Uniform illumination of the palmar region is the result of the employed acquisition system which featured a ring-shaped light source
} 


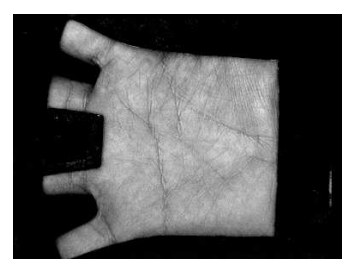

(a)

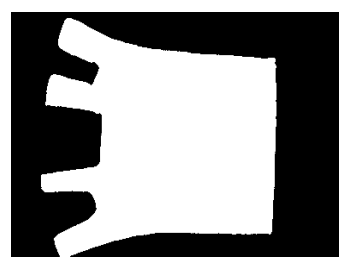

(b)

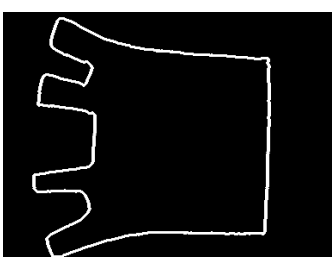

(c)

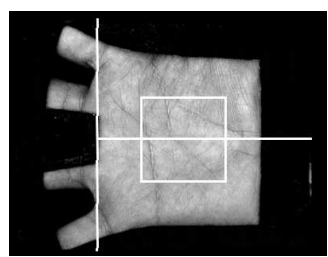

(d)

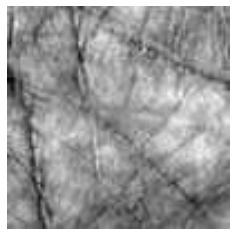

(e)

Figure 7. The pre-processing procedure of the PolyU database: (a) a hand-image from the PolyU database, (b) the binary image of the hand region, (c) the image of the hand region contour, (d) extraction of the palm-print ROI, (e) the normalized palm-print image

\subsection{The experimental setup}

For the verification experiments presented in the next session a closed-set experimental protocol was chosen. For these kinds of experiments the available data has to be partitioned into two non-overlapping groups. The first group represents the client data, while the second group represents the impostor data ${ }^{3}$. In our case both databases were randomly divided in such a way that the client group contained the hand-images of roughly $60 \%$ of the subjects in the database, while the remaining $40 \%$ were assigned to the impostor group. The impostor group was then further partitioned into a group of evaluation impostors and a group of test impostors (in a ratio of $3: 5$, respectively). In the next step the images of the subjects assigned to the client group were also divided into three sets: the training set (three images per subject), the evaluation set (three images per subject) and the test set (the remaining images of the given subject not included in the training or evaluation sets). Images from the client training set were used to construct client-templates (i.e., mean feature vectors), images from the impostor as well as the client evaluation set (or group) were used to compute the decision threshold and to optimize the system parameters (i.e., number of scales and orientations of the 2D log-Gabor filters), while the remaining test sets were employed exclusively for the final performance evaluation. In the experiments with the evaluation and test image sets each of the client images was compared to the corresponding class, i.e., client template, in the database, whereas all impostor images, i.e., 10 for the FE-LUKS and 20 (on average) for the PolyU database, were compared to each of the classes, i.e., client templates, in the database. The described experimental setup is summarized in Table 1 (here the symbol $\ddagger$ denotes that the experiments were performed in the evaluation stage, the symbol $\dagger$ stands for the test stage and $*$ signals that the underlying number denotes the average value - note that there are only approximately 20 images per subject in the PolyU database).

Once all the experiments, i.e., all client and impostor experiments, were performed the verification accuracy of the proposed feature-extraction technique was assessed using three error rates: the false acceptance rate (FAR),

\footnotetext{
${ }^{3} \mathrm{~A}$ client is a subject enrolled in the system, i.e., there is a client-model for that subject in the systems' database, while an impostor is a subject not enrolled in the system, i.e., there is no client-model for that subject in the systems' database
} 
Table 1. The experimental setup for the FE-LUKS and PolyU databases

\begin{tabular}{llllll}
\hline & FE-LUKS & PolyU & & FE-LUKS & PolyU \\
\hline No. of clients & 120 & 230 & No. of test images per client & 4 & $14^{*}$ \\
No. of eval. impostors & 30 & 60 & No. of client experiments & $360^{\ddagger}$ & $690^{\ddagger}$ \\
No. of test impostors & 50 & 96 & No. of impostor experiments & $36000^{\ddagger}$ & $276000^{\ddagger *}$ \\
No. of train. images per client & 3 & 3 & No. of client experiments & $480^{\dagger}$ & $2040^{\dagger *}$ \\
No. of eval. images per client & 3 & 3 & No. of impostor experiments & $60000^{\dagger}$ & $441600^{\dagger *}$ \\
\hline \hline
\end{tabular}

which measures the frequency of falsely accepted impostors, the false rejection rate (FRR), which measures the frequency of falsely rejected clients, and the total error rate (TER), which is defined as TER=FAR+FRR. The values of FAR and FRR, however, depend heavily on the selected decision threshold. Choosing a threshold that ensures a low FRR results in a high value of the FAR and vice versa, the threshold that ensures a low FAR results in a high FRR. To effectively compare different feature extraction techniques (or assess the performance of a given system) a decision threshold has to be selected which ensures some pre-defined values of the FAR and FRR. In this paper three such thresholds (or operating points) were selected, namely, the threshold that ensured FAR=FRR in the evaluation experiments, the threshold that ensured $\mathrm{FAR}=0.1 \mathrm{FRR}$ in the evaluation experiments and finally the threshold that ensured FAR=10FRR in the evaluation experiments. It should, however, be noted that even though the decision thresholds were set using the palm-print data assigned to the evaluation set, the computed thresholds ensured only approximate values of the FAR and FRR at the three operating points in the evaluation experiments, i.e., $F A R \approx F R R, F A R \approx 0.1 F R R$ and $F A R \approx 10 F R R$. Such results are common in the field of biometrics, as the performance evaluation procedure of biometric verification systems is linked to a specific database of limited size and, thus, the available number of client samples usually determines the quantization step for the false rejection rate (which is normally quite large). Or, in other words, a small change in the value of the decision threshold induces a rather large change in the relation of the FAR and FRR. Therefore, the threshold that best approximates the given operating point is selected and used for the final performance assessment on the test set.

\section{The experiments}

In this section, the verification experiments on the two palm-print databases and their results are presented to demonstrate the feasibility of the proposed phase-congruency based feature-extraction technique. 


\subsection{Parameter tuning}

Our first series of verification experiments was performed on the sets of evaluation images of the FE-LUKS and PolyU databases. The experiments aimed at assessing the performance of the proposed feature-extraction technique with respect to the number of scales and orientations of the 2D log-Gabor filters employed for computation of the phase congruency features. The experiments were carried out with five different combinations of the values of $p$ and $r$ (see Section 3.1 for details). In all cases the number of features was set to its maximal value, i.e., $d^{\prime}=119$ for the FE-LUKS and $d^{\prime}=229$ for the PolyU database. In the matching stage the cosine similarity measure was used for matching score calculation. The Receiver Operating Characteristic (ROC) curves, which plot the values of FAR and FRR at various decision thresholds, generated during the experiments are presented in Fig. 8. The actual values of the error rates at the three characteristic operating points of the ROC curves are presented in Table 2. Here the best performing combination of the numbers of filter scales and orientations at a given operating point is presented in bold (for each database).

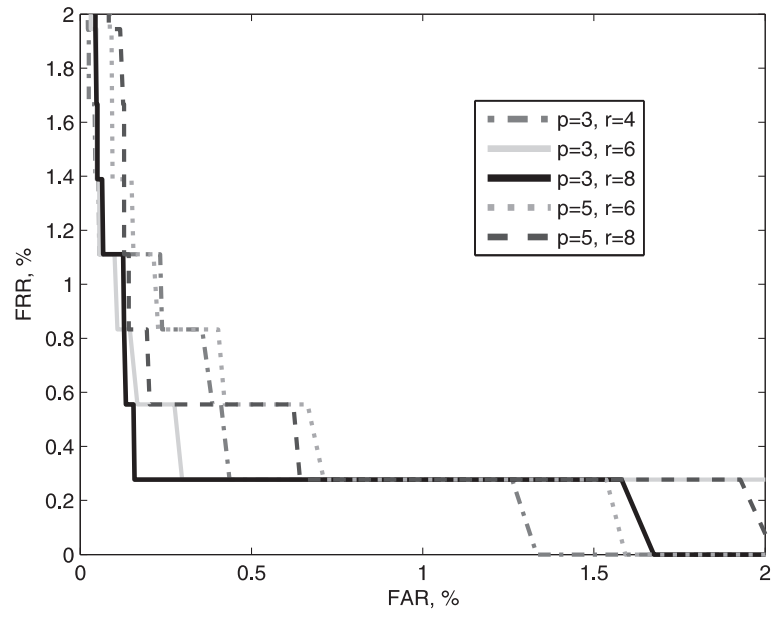

(a)

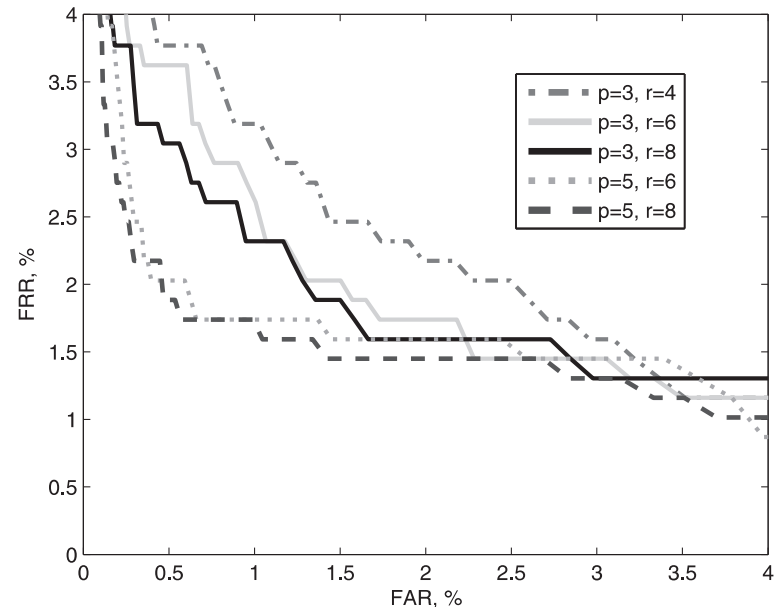

(b)

\section{Figure 8. ROC curves of the verification experiments on the evaluation image sets: (a) for the FE- LUKS palm-print database, (b) for the PolyU palm-print database}

As we can see from the results, none of the tested combinations achieved the best results at all operating points. On the FE-LUKS database the combination of 3 scales and 8 orientations performed the best at the equal error operating point, i.e., at FAR $=\mathrm{FRR}$, while 3 filter scales combined with 4 and 6 orientations resulted in the lowest TER at the remaining two operating points, i.e., at FAR $=10 \mathrm{FRR}$ and $\mathrm{FAR}=0.1 \mathrm{FRR}$, respectively. However, considering the fact that the equal error operating point is the most commonly used error rate when assessing the performance of different biometric systems (or algorithms), 3 filter scales and 8 filter orientations are chosen for the final comparative assessment on the FE-LUKS database presented in the next section. 
Table 2. The error rates FAR, FRR and TER (in \%) from three operating points of the ROC curves. Here NS denotes the number of employed filter scales and NO the number of employed filter orientations.

\begin{tabular}{llllllllllll}
\hline \hline Operating point & & \multicolumn{1}{c}{} & \multicolumn{3}{c}{ FAR=FRR } & \multicolumn{3}{c}{ FAR $=0.1 F R R$} & \multicolumn{3}{c}{ FAR $=10 \mathrm{FRR}$} \\
\hline Database & NS- $p$ & NO- $r$ & FAR & FRR & TER & FAR & FRR & TER & FAR & FRR & TER \\
\hline FE-LUKS & 3 & 4 & 0.41 & 0.56 & 0.97 & 0.11 & 1.11 & 1.22 & 1.26 & 0.28 & $\mathbf{1 . 5 4}$ \\
& 3 & 6 & 0.28 & 0.56 & 0.84 & 0.10 & 1.11 & $\mathbf{1 . 2 1}$ & 2.73 & 0.28 & 3.01 \\
& 3 & 8 & 0.28 & 0.28 & $\mathbf{0 . 5 6}$ & 0.12 & 1.11 & 1.23 & 1.54 & 0.28 & 1.82 \\
& 5 & 6 & 0.54 & 0.56 & 1.10 & 0.13 & 1.39 & 1.52 & 1.58 & 0.28 & 1.86 \\
PolyU & 5 & 8 & 0.54 & 0.56 & 1.10 & 0.13 & 1.39 & 1.52 & 1.93 & 0.28 & 2.21 \\
& 3 & 4 & 2.17 & 2.17 & 4.34 & 0.39 & 4.06 & 4.45 & 10.10 & 1.01 & 11.11 \\
& 3 & 6 & 1.73 & 1.74 & 3.47 & 0.35 & 3.62 & 3.97 & 8.70 & 1.01 & 9.71 \\
& 3 & 8 & 1.58 & 1.74 & 3.32 & 0.31 & 3.19 & 3.50 & 8.37 & 0.87 & 9.24 \\
& 5 & 6 & 1.54 & 1.59 & 3.13 & 0.27 & 2.75 & 3.02 & 6.94 & 0.72 & $\mathbf{7 . 6 6}$ \\
& 5 & 8 & 1.42 & 1.45 & $\mathbf{2 . 8 7}$ & 0.23 & 2.61 & $\mathbf{2 . 8 4}$ & 7.83 & 0.87 & 8.70 \\
\hline \hline
\end{tabular}

For the PolyU database the best results were observed when 2D log-Gabor filters at 5 scales and 8 orientations were used in the feature-extraction stage. In this setting the lowest TER was achieved at two operating points, i.e, $\mathrm{FAR}=\mathrm{FRR}$ and $\mathrm{FAR}=10 \mathrm{FRR}$. Furthermore, we can notice that all error rates were higher than the corresponding ones obtained on the FE-LUKS database. The reason for such behavior can most likely be found in the size of the database. As the PolyU database contains palm-prints of twice as many subjects as the FE-LUKS database, it is harder for the feature-extraction technique to appropriately discriminate between different subjects.

\subsection{Performance assessment}

The goal of the second series of our verification experiments was to assess the accuracy of the proposed approach on an independent set of test images. To that end, our phase congruency based feature extraction technique was implemented with 3 filter scales and 8 filter orientations for the FE-LUKS database and 2D log-Gabor filters at 5 scales and 8 orientations for the PolyU database. However, as the final error rates estimated in the performance assessment depend heavily on the choice of the images used for training and testing, all experiments presented in this section were repeated 5 times. Each time the subjects of both databases were split randomly into the client and impostor groups and their images were then distributed amongst the training, evaluation and test image sets - of course in accordance with the protocol described in Section 5.3. Thus, more than 300000 verification attempts were performed on the FE-LUKS database and more than 2 million on the PolyU database. In each repetition the thresholds pre-set on the evaluation data were used in the experiments. Such an approach ensured real life settings where the decision threshold for a specific operating point is determined in advance and the final performance assessment aims at: (i) evaluating the error rates on unseen data which has not been used for tuning the system's 
parameters, and (ii) examining the calibration of the system, i.e., examining how much the final error rates deviate from the operating point at which the employed decision threshold was set.

In addition to the proposed approach, four popular palm-print feature-extraction techniques were also implemented, trained and compared to our approach, namely, the Eigenpalm approach [14], the Fisherpalm approach [13], the line-feature [10] based approach (denoted as LFBA in Table 3) in combination with $\mathrm{LDA}^{4}$ and the palmprint orientation code (POC) technique [11]. The cosine similarity measure was used as the scoring function for all methods except for the POC technique were the Hamming distance was found to be more appropriate.

The results of the experiments in terms of the average FAR, FRR and TER (over five repetitions of the verification experiments) for the three operating points are presented in Table 3 - the lowest error rates at each operating point are again presented in bold. The proposed line features resulted in the best verification performance on the FE-LUKS database and outperformed the other two line-feature based methods, i.e., the LFBA and the POC technique, on the PolyU database. The only approach achieving even lower error rates than the proposed technique on the PolyU database was the Fisherpalm approach. To test the statistical significance of our results, several paired-samples T-tests (with a significance level of $\alpha=0.05$ ) were performed on the TERs obtained at the equal error operating point of each of the five experimental repetitions. The tests showed that there is a significant reduction in the mean TER with the $p$-value (or significance) of 0.047 and 0.016 when our method was compared to the Fisherpalm and LFBA technique on the FE-LUKS database, while the remaining tests on the FE-LUKS database resulted in $p$-values even lower than $10^{-4}$. For the PolyU database the tests showed a similar setting: the Fisherpalm technique significantly outperformed the proposed approach with the $p$-value of $2.8 \cdot 10^{-5}$, while the phase congruency technique led to a significant decrease in the mean TERs at the equal error operating point in comparison to the LFBA, Eigenface and POC techniques with the corresponding $p$-values being 0.008, 0.001 and $6.3 \cdot 10^{-5}$, respectively. Note again that the equal error operating point was chosen for the significance testing because it represents the most common operating point at which the error rates of biometric systems are reported in the literature.

While the proposed phase congruency approach resulted in the best verification performance on the FE-LUKS database at two different operating points, the Fisherpalm approach significantly out-performed the proposed technique on the PolyU database at all operating points. The reason for such behavior could be found in the block-wise smoothing (via mean and standard deviation calculation) of the phase-congruency image, which might have removed too much detail and consequently resulted in a loss of discriminative information. In any case, it represents an interesting issue which will be analyzed in detail in our future work. Based on the presented results we can conclude that the proposed technique is suitable for verifying identity claims based on palm-print images

\footnotetext{
${ }^{4}$ Note that the original LFBA as presented in [10] does not use LDA to extract the final palm-print features. LDA was added to allow for a fair comparison with the proposed approach which also includes a LDA step.
} 
Table 3. Average error rates FAR, FRR and TER (in \%) obtained with three thresholds set at three different operating points of the ROC curves generated during the evaluation phase.

\begin{tabular}{lllllllllll}
\hline \hline Operating point & & \multicolumn{3}{c}{ FAR=FRR } & \multicolumn{3}{c}{ FAR $=0.1$ FRR } & \multicolumn{3}{c}{ FAR $=10$ FRR } \\
\hline Database & Method & FAR & FRR & TER & FAR & FRR & TER & FAR & FRR & TER \\
\hline FE-LUKS & Eigenpalm & 2.42 & 2.67 & 5.09 & 8.79 & 0.92 & 9.71 & 0.75 & 6.33 & 7.08 \\
& Fisherpalm & 0.98 & 0.63 & 1.61 & 3.15 & 0.33 & $\mathbf{3 . 4 8}$ & 0.23 & 2.00 & 2.23 \\
& LFBA & 1.35 & 0.83 & 2.18 & 5.34 & 0.13 & 5.47 & 0.36 & 2.83 & 3.19 \\
& POC & 2.05 & 1.75 & 3.80 & 8.62 & 0.50 & 9.12 & 0.39 & 4.29 & 4.68 \\
& Proposed & 0.73 & 0.63 & $\mathbf{1 . 3 6}$ & 3.27 & 0.29 & 3.56 & 0.19 & 1.58 & $\mathbf{1 . 7 7}$ \\
PolyU & Eigenpalm & 2.33 & 2.10 & 4.43 & 8.78 & 0.75 & 9.53 & 0.55 & 4.73 & 5.28 \\
& Fisherpalm & 1.15 & 1.21 & $\mathbf{2 . 3 6}$ & 5.22 & 0.53 & $\mathbf{5 . 7 5}$ & 0.22 & 2.26 & $\mathbf{2 . 4 8}$ \\
& LFBA & 2.08 & 1.98 & 4.06 & 9.17 & 1.06 & 10.23 & 0.39 & 3.68 & 4.07 \\
& POC & 11.48 & 11.69 & 23.17 & 44.44 & 4.38 & 48.82 & 2.08 & 21.31 & 23.39 \\
& Proposed & 1.58 & 1.76 & 3.34 & 7.64 & 0.90 & 8.54 & 0.30 & 3.01 & 3.31 \\
\hline \hline
\end{tabular}

with distortions, while it still achieves relatively low error rates when high quality palm-print images are used for verification.

\section{Conclusion}

We have presented a novel approach to palm-print verification that uses phase congruency and linear discriminant analysis for the extraction of discriminative palm-print features. The feasibility of the proposed approach was demonstrated in a series of verification experiments performed on two databases with contrasting characteristics, namely, the FE-LUKS and the PolyU databases. The experimental results suggest that the proposed approach outperforms popular feature-extraction schemes such as the Eigenpalm, the Fisherpalm or the Palm-print Orientation Code techniques when identity claims have to be verified based on palm-print images with distortions, i.e., acquired with a scanner (FE-LUKS), while still achieving reasonable verification performance when high-quality palm-print images, i.e., captured with a CCD camera (PolyU), are employed for verification. Based on these encouraging results, our future work will be focused on a more detailed analysis of the behavior of the phase congruency model in comparison with other established techniques, such as the Fisherpalm approach, as well as on exploring the possibilities of integrating phase congruency features into multi-modal, i.e., intra-modal, palm-print recognition systems and investigating other options for robust line (or edge) feature extraction (for example the one presented in [30]), which could improve upon the presented phase-congruency model. 


\section{Acknowledgements}

This work has been partially supported by the national research program P2-0250(C) Metrology and Biometric Systems and by the European Commission under contract FP7-217762 HIDE. Homeland security, biometric identification and personal detection ethics.

\section{References}

[1] Dorizzi, B., and Ariyaeeinia, A.: 'A Proposal for structured activities in biometric identity authentication'. In: Proc. of the COST Workshop on the Advent of Biometrics on the Internet, 2002, pp. 3-8

[2] Jain, A.K., Ross, A., and Prabhakar, S.: 'An Introduction to Biometric Recognition'. IEEE Transactions on Circuits and Systems for Video Technology, 2004, 14, (1), pp. 4-20

[3] Ribarić, S., Ribarić, D., and Pavešić, N.: 'Multimodal Biometric User-identification System for Network-based Applications'. IEE Proceedings - Vision, Image and Signal Processing, 2003, 150, (6), pp. 409-416

[4] Pavešić, N., Ribarić, S., and Ribarić, D.: 'Personal Authentication Using Hand-Geometry and Palmprint Features - The State of the Art'. Proce. of the Workshop: Biometrics - Challenges Arising from Theory to Practice, Cambridge, 2004, pp. 17-26.

[5] Yoruk, E., Dutagaci, H., and Sankur, B.: 'Hand Biometrics'. Image and Vision Computing, 2006, 24,(5), pp. 483-497

[6] Kumar, A., and Zhang, D.: 'Personal Authentication Using Multiple Palmprint Representation'. Pattern Recognition, 2005, 38, (10), pp. 1695-1704

[7] Zhang, D., Kong, W.K., You, J., and Wong, M.: 'Online Palmprint Identification'. IEEE Transactions on Pattern Analysis and Machine Intelligence, 2003, 25, (9), pp. 1041-1050

[8] Wu, X.Q., Wang, K.Q., and Zhang, D.: 'Wavelet Based Palmprint Recognition', Proc. of Int. Conf. Machine Learning and Cybernetics, Beijing, 2002, pp. 1253-1257

[9] Zhang, D., and Shu, W.: 'Two Novel Characteristics in Palmprint Verification: Datum Point Invariance and Line Feature Matching'. Pattern Recognition, 1999, 32, (4), pp. 691-702

[10] Kumar, A., Wong, D.C.M., Shen, H.C., and Jain, A.K.: 'Personal Authentication Using Hand Images'. Pattern Recognition Letters, 2006, 27, (13), pp. 1478-1486 
[11] Wu, X., Wang, K., and Zhang, D.: 'Palmprint Authentication Based on Orientation Code Matching'. AVBPA 2005, Lecture Notes in Computer Science 3546, Springer, 2005, pp. 555-562

[12] Huang, D.S., Jia, W., and Zhang, D.: 'Palmprint Verification Based on Principal Lines'. Pattern Recognition, 2008, 41, (4), pp. 1316-1328

[13] Wu, X., Zhang, D., and Wang, K.: 'Fisherpalms Based Palmprint Recognition'. Pattern Recognition Letters 2003, 24, (15), pp. 2829-2838

[14] Lu, G., Zhang, D., and Wang, K.: 'Palmprint Recognition Using Eigenpalm Features'. Pattern Recognition Letters 2003, 24, (9-10), pp. 1463-1467

[15] Shang, L., Hunag, D.S., Du, J.X., and Zheng, C.H.: 'Palmprint Recognition Using FastICA Algorithm and Radial Basis Probabilistic Neural Network'. Neurocomputing, 2006, 69, (13-15), pp. 1782-1786

[16] Hu, D., Feng, G., and Zhou, Z.: 'Two-dimensional Locality Preserving Projections (2DLPP) With its Application to Palmprint Recognition'. Pattern Recognition, 2007, 40, (1), pp. 339-342

[17] Savič, T., and Pavešić, N.: 'Personal Recognition Based on an Image of the Palmar Surface of the Hand'. Pattern Reognition, 2007, 40, (11), pp. 3152-3163

[18] Pavešić, N., Savič, T., Ribarić, S., and Fratrić, I.: 'A Multimodal Hand-based Verification System With an Aliveness-detection Module'. Annals of Telecommunications, 2007, 62, (1-2), pp. 3152-3163

[19] Han, C.C., Cheng, H.L., Lin, C.L., and Fan, K.C.: 'Personal Authentication Using Palm-print Features'. Pattern recognition, 2003, 36, (2), pp. 371-381

[20] Kovesi, P.: 'Image Features From Phase Congruency'. Videre: Journal of Computer Vision Research 1999, 1, (3), pp. $1-26$

[21] Gundimada, S., and Asari, V.K.: 'A Novel Neighborhood Defined Feature Selection on Phase Congruency Images for Recognition of Faces with Extreme Variations'. International Journal of Information Technology, 2006, 3, (1), pp. 25-31

[22] Duda, R., and Hart, P.: 'Pattern Classification and Scene Analysis' (John Wiley and Sons, 1973), pp. 267-272

[23] Marr, D., and Hildreth, E.C.: 'Theory of Edge Detection'. Proceedings of the Royal Society of London, Series B, 1980, 207, pp. 187-217

[24] Canny, J.F.: 'A Computational Approach to Edge Detection'. IEEE Transactions on Pattern Analysis and Machine Intelligence, 1986, 8, (6), pp. 112-131 
[25] Štruc, V., Mihelič, F., and Pavešić, N.: 'Face Authentication Using a Hybrid Approach', Journal of Electronic Imaging, 2008, 17, (1), doi. 011003

[26] Žibert, J., Vesnicer, B., and Mihelič, F.: 'Development of a Speaker Diarization System for Speaker Tracking in Audio Broadcast News: a Case Study'. Journal of Computing and Information Technology - CIT, 2008, 16, (3), pp. 183-195

[27] Ribarić, S., and Fratrić, I.: 'A Biometric Identification System Based on Eigenpalm and Eigenfinger Features'. IEEE Transactions on Pattern Analysis and Machine Intelligence, 2005, 27, (11), pp. 1698-1709

[28] Wong, M., Zhang, D., Kong, W.K., and Lu, G.: 'Real-time Palmprint Acquisition System Design'. IEE Proceedings - Vision, Image and Signal Processing, 2005, 152, (5), pp. 527-534

[29] The PolyU Palmprint Database, http://www4.comp.polyu.edu.hk/ biometrics/, accessed August 2008

[30] Konishi, S., Yuille, A.L., Coughlan, J.M., and Zhu, S.C.: 'Statistical Edge Detection: Learning and Evaluating Edge Cues'. IEEE Transactions on Pattern Analysis and Machine Intelligence, 2003, 25, (1), pp. 57-74 